\title{
Análise da realização da cirurgia ambulatorial na perspectiva da qualificação e resolutividade do cuidado prestado pelo médico de família e comunidade na Atenção Primária à Saúde na cidade do Rio de Janeiro
}

\author{
Analysis of the performance of ambulatory surgery in primary health care by the family physician \\ from the perspective of qualification and resolution of care in the city of Rio de Janeiro
}

\section{Análisis de la realización de la cirugía ambulatoria en la atención primaria a la salud en la perspectiva de la calificación y resolución del cuidado en la ciudad de Rio de Janeiro}

\author{
Philipp Rosa Oliveira ${ }^{1} \mathbb{D}$, Cesar Augusto Orazem Favoreto $^{2}(\mathbb{D}$ \\ ${ }^{1}$ Secretátia Municipal de Saúde do Rio de Janeiro. Rio de Janeiro, RJ, Brasil. \\ 2 Universidade Estadual do Rio de Janeiro (UERJ). Rio de Janeiro, RJ, Brasil.
}

\section{Resumo}

Introdução: A Estratégia de Saúde da Família, a partir de 1998, representa o modelo de organização e de qualificação da Atenção Primária à Saúde no Brasil. No município do Rio de Janeiro, nos últimos anos, ocorreu um incremento rápido na cobertura da população pelas equipes de saúde da família, com a perspectiva de desenvolvimento de uma Atenção Primária à Saúde abrangente. Nesta perspectiva surge a cirurgia ambulatorial, que tem sido incorporada muito timidamente às práticas das Unidades Básicas. Objetivos: Este artigo analisa a incorporação da cirurgia ambulatorial pelas Equipes de Saúde da Família como um dos aspectos envolvidos na ampliação da coordenação do cuidado e da resolutividade da Atenção Primária à Saúde. Métodos: Foi realizado um estudo exploratório descritivo, utilizando-se da análise dos dados da produção dos procedimentos cirúrgicos na rede de atenção básica da cidade. O estudo analisou as informações disponíveis no banco de dados denominado Carteirômetro no período de outubro de 2015 a março de 2017, além de entrevistas online com médicos de família atuantes na Estratégia de Saúde da Família. A expansão das equipes de saúde da família neste período foi acompanhada do aumento percentual dos procedimentos de cirurgia ambulatorial (63,8\%). Resultados: Contudo, esta evolução representa, ainda, um tímido quantitativo de cirurgias realizadas pelo número de equipes implantadas, ou seja, cerca de 1,5 procedimento/equipe no último trimestre pesquisado. Conclusão: Entre os fatores limitadores destas intervenções, identificou-se a ausência de capacitação profissional para a execução destes procedimentos, além de estrutura física e processual inadequada das unidades de saúde.

Palavras-chave: Atenção Primária à Saúde; Estratégia Saúde da Família; Procedimentos Cirúrgicos Ambulatórios

Como citar: Oliveira PR, Favoreto CAO. Análise da realização da cirurgia ambulatorial na perspectiva da qualificação e resolutividade do cuidado prestado pelo médico de família e comunidade na Atenção Primária à Saúde na cidade do Rio de Janeiro. Rev Bras Med Fam Comunidade. 2019;14(41):1864 http://dx.doi.org/10.5712/rbmfc14(41)1864

\author{
Autor correspondente: \\ Philipp Rosa Oliveira. \\ E-mail: oliveiraphilipp@gmail.com \\ Fonte de financiamento: \\ declaram não haver. \\ Parecer CEP: \\ 2.270.511 (SMS/RJ), aprovado em \\ 13/09/2017; 1.961 .357 (UFRJ), aprovado \\ em 13/03/2017.
}

Procedência e revisão por pares: revisado por pares.

Recebido em: 30/08/2018.

Aprovado em: 08/01/2019. 


\begin{abstract}
Introduction: The Family Health Program was created in 1994 and was reoriented as a strategy to change the care model from 1998 onwards, becoming a model for the organization and qualification of Primary Health Care in Brazil. In Rio de Janeiro city, in recent years, there has been a rapid increase in the coverage of the population by the family health teams, with the perspective of developing comprehensive Primary Health Care. In this perspective emerges the outpatient surgery, which has been incorporated very timidly to the practices of the Basic Health Units. Objectives: This article analyzes the incorporation of outpatient surgery by the Family Health Teams as one of the aspects involved in the expansion of the coordination of care and the resolution of Primary Health Care in this municipality. Methods: A descriptive exploratory study was carried out using the data analysis of the production of surgical procedures in the basic care network of the city. The study analyzed the information available on the Carteirômetro database for the period of October 2015 to March 2017, in addition to online interviews with family doctors working in the Family Health Strategy. It was observed that the expansion of the Family Health Strategy teams during the study period was accompanied by the percentage increase in outpatient surgery procedures (63.8\%). Results: However, this evolution also represents a timid amount of surgeries performed by the number of teams implanted, that is, about 1.5 procedure/team in the last quarter surveyed. Conclusion: Among the limiting factors for the development of these interventions in Primary Health Care was the lack of professional training for the execution of these procedures, as well as inadequate physical and procedural structure of the health units.
\end{abstract}

Keywords: Primary Health Care; Family Health Strategy; Ambulatory Surgical Procedures

\title{
Resumen
}

Introducción: La estrategia de Salud de la Familia, a partir de 1998, modelo de organización y de calificación de la Atención Primaria a la Salud en Brasil. En la ciudad de Rio de Janeiro, en los últimos años, ocurrió un rápido incremento en la cobertura de la población por los equipos de salud de la familia, con la perspectiva de desarrollar una Atención Primaria a la Salud integral. En esta perspectiva surge la cirugía ambulatoria, que ha sido incorporada muy tímidamente a las prácticas de las Unidades Básicas de Salud. Objetivos: Este artículo analiza la incorporación de la cirugía ambulatoria por los Equipos de Salud de la Familia como uno de los aspectos involucrados en la ampliación de la coordinación del cuidado y de la resolutividad de la Atención Primaria a la Salud en este municipio. Métodos: Se realizó un estudio exploratorio descriptivo, utilizando el análisis de los datos de la producción de los procedimientos quirúrgicos en la red de atención básica de la ciudad. El estudio analizó la información disponible en la base de datos Carteirômetro y Tabnet referente al período de octubre de 2015 a marzo de 2017, además de entrevistas en línea con médicos de familia actuantes en la Estrategia de Salud de la Familia. Se observó que la expansión de los equipos de la Estrategia de Salud de la Familia durante el período de estudio fue acompañada del aumento porcentual de los procedimientos de cirugía ambulatoria (63,8\%). Resultados: Sin embargo, esta evolución representa, además, un tímido cuantitativo de cirugías realizadas por el número de equipos implantados, o sea, cerca de 1,5 procedimiento/equipo en el último trimestre investigado. Conclusión: Entre los factores limitadores del desarrollo de estas intervenciones en la Atención Primaria a la Salud se identificó la ausencia de capacitación profesional para la ejecución de estos procedimientos, además de estructura física y procesal inadecuada de las unidades de salud.

Palabras clave: Atención Primaria de Salud; Estratégia de Salud Familiar; Procedimientos Quirúrgicos Ambulatorios

\section{Introdução}

O Programa de Saúde da Família (PSF) surgiu em 1994 com a proposta de criar uma Atenção Primária à Saúde (APS) seletiva, ou seja, voltada para grupos populacionais específicos, focalizados a uma população muito empobrecida, com serviços restritos e com baixa articulação com o restante da rede de atenção à saúde. ${ }^{1}$ Em 1998, o PSF deu lugar à Estratégia de Saúde da Família (ESF), proposta como forma de reestruturação do modelo assistencial do Sistema Único de Saúde (SUS), por meio da organização e aplicação de atributos que garantissem a qualidade da atenção prestada. A Política Nacional de Atenção Básica, de 2006, explicita a importância de construção de uma APS abrangente, integral e organizadora da rede assistencial, de modo a evoluir no desenvolvimento dos princípios e diretrizes do SUS. ${ }^{2}$

De acordo com Mendes, ${ }^{3}$ a atenção primária resolutiva, integral como elemento central de uma Rede de Atenção à Saúde (RAS) pode ser capaz de ampliar a resolutividade da atenção prestada pela rede, aumentar sua efetividade, racionalizar seus custos e ser capaz de promover uma atenção mais integral à saúde da população. 
Esta maior resolutividade da ESF pode reduzir a utilização excessiva e descoordenada da atenção especializada, evitar a formação de longas filas de espera, diminuir os custos tanto para os pacientes como para o sistema e, muitas vezes, pode evitar desfechos indesejados. O processo de ampliar e qualificar a atenção pela ESF implica em novas concepções sobre o processo de trabalho, o desenvolvimento de novas competências profissionais e de condições operacionais e estruturais das unidades de saúde para a incorporação de novas intervenções terapêuticas e diagnósticas nas práticas das equipes. ${ }^{4}$

Entre estas intervenções, o foco deste trabalho é a introdução e operacionalização de pequenos procedimentos de cirurgia ambulatorial pelas equipes de saúde da família no cenário da rede básica no município do Rio de Janeiro. O objeto e os objetivos estão inseridos, portanto, na perspectiva de ampliação da resolutividade, tendo como pano de fundo a integralidade, acesso e vínculo/coordenação do cuidado. ${ }^{5}$

Em sistemas de saúde com APS pouco resolutiva, os pacientes encontram dificuldade de acesso a estes tipos de intervenção e, de modo geral, são dirigidos a serviços especializados de nível secundário, ou mesmo serviços hospitalares. As referências aos serviços especializados podem causar dificuldades de acesso e de acessibilidade aos pacientes devido às filas de espera, diferenças de fluxos assistenciais entre os serviços e deslocamento para áreas distantes das moradias.

Na cidade ocorreu uma forte expansão da ESF. A cobertura populacional passou de 3,5\%, em 2008, para cerca de $70 \%$, no final de $2016 .{ }^{5}$ Foram feitos investimentos na criação e qualificação das unidades de saúde, expansão da residência em medicina de família e comunidade e de enfermagem na atenção primária, bem como na ampliação e reorientação da regulação municipal por meio do Sistema de Regulação (SISREG). ${ }^{6}$

No momento da pesquisa (2016/2017) existiam implementadas 1.263 equipes de Estratégia de Saúde da Família completas. ${ }^{6}$ Na perspectiva de garantir, avaliar e regular a realização de um conjunto de ações de promoção, prevenção, atenção/recuperação em saúde que unformizasse a atenção prestada na rede de atenção primária da cidade foi formulada uma Carteira de Serviços. A carteira representou uma forma de induzir a incorporação de novas intervenções diagnósticas e terapêuticas, entre elas a realização de pequenos procedimentos em cirurgia ambulatorial. ${ }^{7}$

O potencial da incorporação das pequenas cirurgias nas unidades básicas de saúde (UBS) seria o de reduzir a pressão de demanda por encaminhamentos para serviços de cirurgia e dermatologia e, consequentemente, otimizar os recursos especializados na rede de atenção do município. Por outro lado, havia a possibilidade de potencializar outros aspectos da atenção prestada pelas equipes como o acesso, a longitudinalidade, o vínculo e a valorização da ESF e, em particular, dos médicos de família e comunidade.

Busca na literatura nacional e internacional sobre cirurgia ambulatorial na APS, nas bases do Scielo, Cochrane, Medline, Lilacs e da Coordenação de Aperfeiçoamento de Pessoal de Nível Superior (CAPES), com a combinação dos descritores procedimentos cirúrgicos ambulatoriais, atenção primária à saúde e estratégia de saúde da família. A pesquisa revelou poucas publicações sobre este tema e controvérsias em relação às avaliações de custo-efetividade destes procedimentos, principalmente quando colocados à luz de uma APS abrangente.

Em uma primeira análise da abrangência sobre o acesso à cirurgia ambulatorial no âmbito da APS, verificou-se que em agosto de 2016 aguardavam atendimento no SISREG, há oito meses, um total de 
2.315 solicitações para pequenos procedimentos dermatológicos. ${ }^{8}$ Eram solicitações para a abordagem de lipoma, cisto epidérmico, nevos, biópsia de pele e remoção de lesões suspeitas de pele, procedimentos que poderiam ser realizados na APS e, consequentemente, evitar o encaminhamento para o setor secundário.

A existência desta demanda reprimida reforçou a importância de analisar o acesso, as condições estruturais e processuais, assim como as repercursões para usuários, serviços, equipes e médicos de familia e comunidade da realização destes procedimentos na ESF.

A pesquisa, portanto, buscou identificar as implicações da incorporação da cirurgia ambulatorial pela ESF no cenário municipal. Esta identificação compreendeu a análise dos dados secundários sobre a realização destes procedimentos na rede local, assim como o questionamento aos médicos das equipes sobre os aspectos estruturais e processuais existentes para efetivar esta prática nas unidades de saúde e suas percepções sobre o impacto destas atividades no cotidiano de suas equipes e na relação com os usuários.

\section{Métodos}

Nesse artigo foi realizado um estudo exploratório e descritivo ${ }^{9}$ sobre a incorporação dos procedimentos cirúrgicos no contexto da ESF no município do Rio de Janeiro e aplicação de um questionário online, com questões fechadas e abertas sobre as condições e percepções dos médicos de família quanto à incorporação da cirurgia ambulatorial em suas práticas.

A delimitação de pequenos procedimentos em cirurgia ambulatorial na atenção primária foi realizada com base nas definições do Caderno de Atenção Básica, número 30, do Ministério da Saúde ${ }^{10}$ que define os procedimentos clínicos e cirúrgicos que devem ser realizados nas diversas unidades de saúde no território brasileiro a partir de protocolos bem estabelecidos. Outro referencial foi a Carteira de Serviços da Secretaria Municipal de Saúde do Rio de Janeiro (SMSRJ), ${ }^{7}$ que dispõe sobre as intervenções a serem realizadas pelas equipes locais. Também foi utilizado o currículo baseado em competências da Sociedade Brasileira de Medicina de Família e Comunidade, voltadas à formação de médicos especialistas em medicina de família e comunidade. ${ }^{11}$

Partindo da heterogeneidade nos objetivos e critérios adotados nestas referências, foi necessário elencar como características para cirurgia ambulatorial na APS as intervenções limitadas à pele, ao tecido celular subcutâneo e anexo, e realizadas sob anestesia locorregional, envolvendo a utilização de materiais estéreis e sala apropriada.

No estudo os parâmetros sobre as condições físicas das unidades de saúde também seguiram as recomendações do Caderno de Atenção Básica, número $30,{ }^{10}$ que preconizam: sala apropriada para a realização dos procedimentos, instrumental cirúrgico mínimo, materiais de proteção individual e de curativos, assim como fluxo para esterilização do material e realização do histopatológico.

Foram extraídas informações dos procedimentos cirúrgicos referentes ao período de outubro 2015 a março de 2017 a partir do Carteirometro. ${ }^{7}$ Este é um banco exstente na plataforma da Subsecretaria de Atenção Primária e Vigilância em Saúde (SUBPAV/RJ), ${ }^{12}$ estruturado com base na carteira de serviços municipal, que armazena dados específicos sobre os procedimentos executados na atenção primária municipal, registrados pelos profissionais nos prontuários eletrônicos. 
Estes dados possibilitaram avaliar a relação entre a implementação da cirurgia ambulatorial e a expansão da ESF na cidade. Os itens do Carteirometro que foram contabilizados foram os procedimentos de sutura, drenagem de abscesso e cantoplastia.

Para a analisar as condições de execução e as percepções dos médicos sobre a incorporação da cirurgia ambulatorial, foi aplicado um questionário online para todos os médicos que atuavam na ESF no Rio de Janeiro há, pelo menos, seis meses, independentemente de serem ou não especialistas em medicina de família ou de realizarem ou não procedimentos cirúrgicos.

O questionário online permite coletar informações com rapidez, sem as limitações da distância. ${ }^{11} \mathrm{O}$ instrumento foi construído através do Google Docs, uma ferramenta de fácil manuseio e entendimento que possibilitou uma coleta de dados.

Os contatos eletrônicos dos médicos foram disponibilizados pela SMSRJ. A todos foi enviada a solicitação de preenchimento e reenviada no total de quatro e-mails no período entre maio e agosto de 2017. O universo de participantes abrangeu 1.263 médicos das equipes. A literatura sobre questionários online relata a existência de dificuldades inerentes a este tipo de coleta eletrônica. Por este motivo, coloca como índice esperado de respostas a estimativa em torno de $7 \%$ do universo da pesquisa. ${ }^{13}$

$O$ instrumento a ser respondido constava de seis questões fechadas que abordavam a realização ou não de cirurgias, os tipos de procedimentos, as condições físicas e instrumentais disponíveis, as oportunidades de capacitações após a graduação, por último, o fluxo e processo de trabalho das equipes para viabilizar o acesso a estas intervenções.

As últimas três perguntas buscaram a percepção dos entrevistados sobre questões subjetivas relacionadas à incorporação das cirurgias nas ações da ESF. Eram, respectivamente, relativas às repercursões destas práticas na visão e no vínculo dos usuários para com as unidades de saúde, equipes e médicos de família e o impacto que percebinham na rede local de atenção à saúde.

O projeto foi aprovado pelo Comitê de Ética em Pesquisa da Escola de Enfermagem Ana Nery, da Universidade Federal do Rio de Janeiro, e pela coparticipante, a SMSRJ, com Certificado de Apresentação para Apreciação Ética (CAAE) de número 63577516.0.3001.5279.

Avaliação das perguntas fechadas foi realizada por meio de análise de frequência simples dos itens respondidos e o cruzamento bivariado de algumas variáveis pela transferência dos dados para uma planilha Excel. Nas três perguntas abertas as respostas foram coletadas e reunidas na forma de pequenos textos que, após uma leitura flutuante, foram agrupados por temas e interpretados em seus sentidos, seguindo-se, assim, a perspectiva de análise de conteúdo proposta por Bardin. ${ }^{14}$

\section{Resultados}

A expansão das ESF no município ocorreu de modo progressivo desde o ano de 2008. No período estudado de 18 meses (de outubro/2015 a março/2017) o incremento foi de 39,25\%, saindo de 930 para 1.295 equipes, alcançando a cobertura de $70,26 \%$ da população.

Os procedimentos aferidos do Carteirômetro foram sutura, drenagem de abscesso e cantoplastia. O termo/registro sutura é genérico e pode ser adotado pelos profissionais em situações diversas desde a excisão e sutura de lesões de pele suspeitas de neoplasia, até abordagem de lesões acidentais. 
Quando analisado o número total destes procedimentos realizados em relação ao número de equipes implantadas, observa-se que, conforme o Gráfico 1 demonstra, houve um aumento em termos absolutos de $63,8 \%$, superior, portanto, aos $39,25 \%$ correspondente à ampliação do número de equipes ESF.

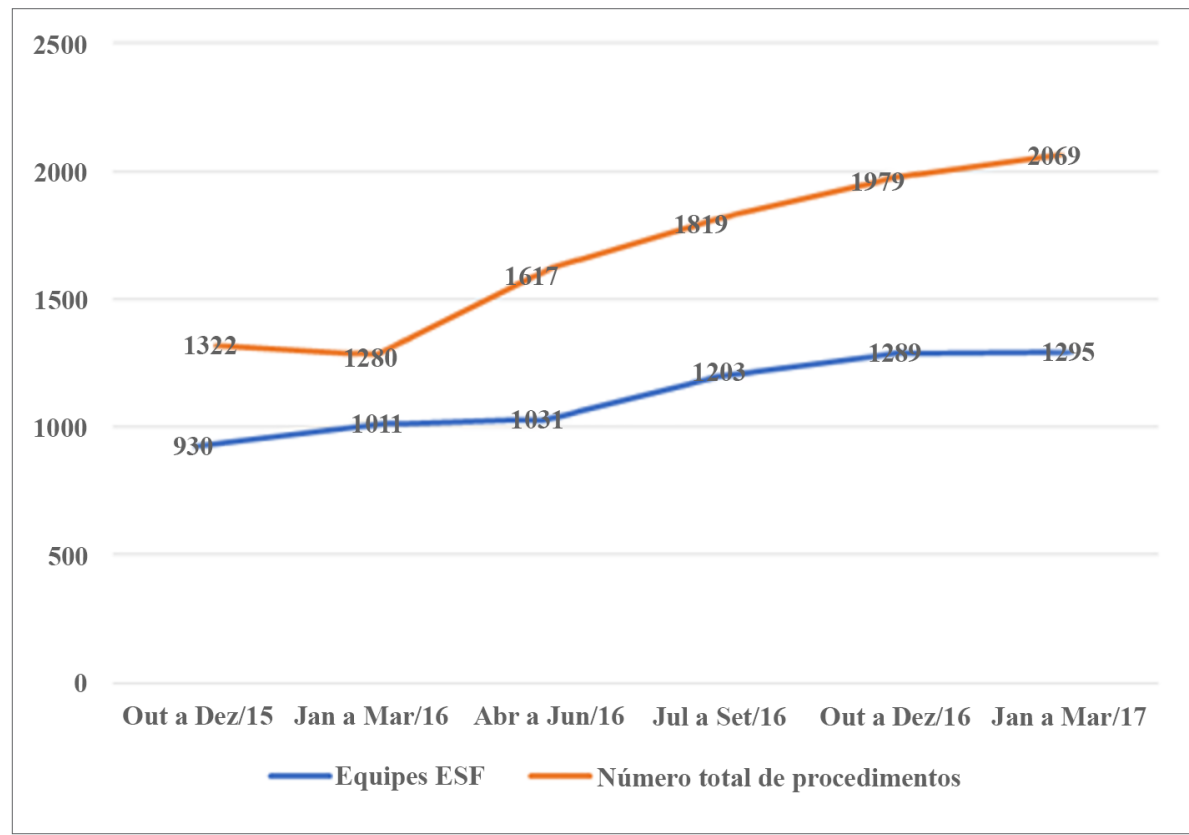

Gráfico 1. Evolução dos registros de todos os procedimentos considerados de cirurgia ambulatorial no Carteirômetro em relação à implantação do número equipes ESF por trimestre. Fonte. Carteirometro/SUBPAV, 2017. Org. Oliveira, 2017.

O número de suturas realizadas no período teve ampliação de 49,3\%. Já os dados sobre a drenagem de abscessos e cantoplastia não demonstram um incremento tão significativo (aumento de 13,7 e 10,3, respectivamente). No Gráfico 2 estão dispostos a distribuição do total de registro de suturas e o número de equipes ESF por trimestre.

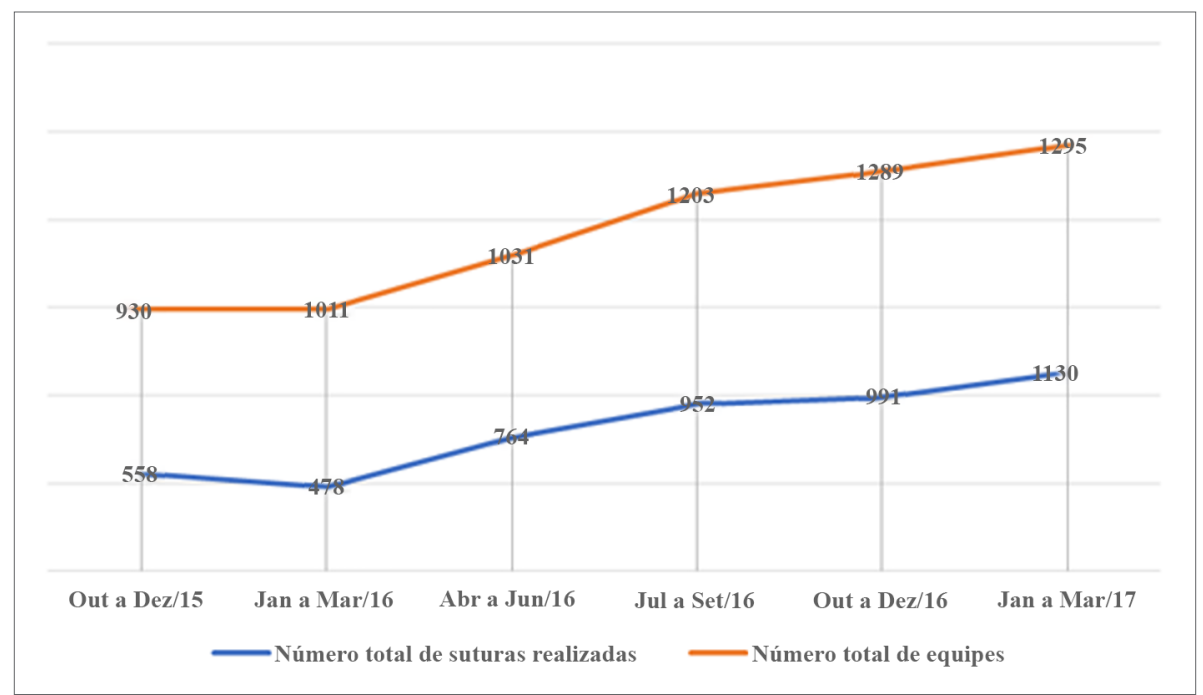

Gráfico 2. Distribuição do número absoluto de registros de suturas existente no Carteirômetro e o numero de equipes ESF por trimestre.

Fonte. Carteirometro/SUBPAV ${ }^{9}$, 2017. Org. Oliveira, 2017. 
A evolução da relação de total de procedimentos por equipe se manteve estável no período (de 1,4 a 1,5/equipe). Já a relação entre número de suturas por equipe teve um discreto aumento de 0,6 para 0,9 em março de 2017. Este resultado pode refletir uma expansão intensa e rápida do número de equipes sem a necessária qualificação dos médicos, dos processos de trabalho e do suporte das unidades de saúde para realizar os procedimentos cirúrgicos.

Importante notar que os números absolutos de drenagem de abscessos (Gráfico 3) não acompanham o aumento observado de informações sobre as suturas. Este resultado pode estar associado à forma como os procedimentos são registrados no prontuário eletrônico, ao fato de serem situações agudas e o atendimento pode ser dirigido a unidades de urgência ou por haver insuficiente estrutura nas unidades para realizar estas intervenções.

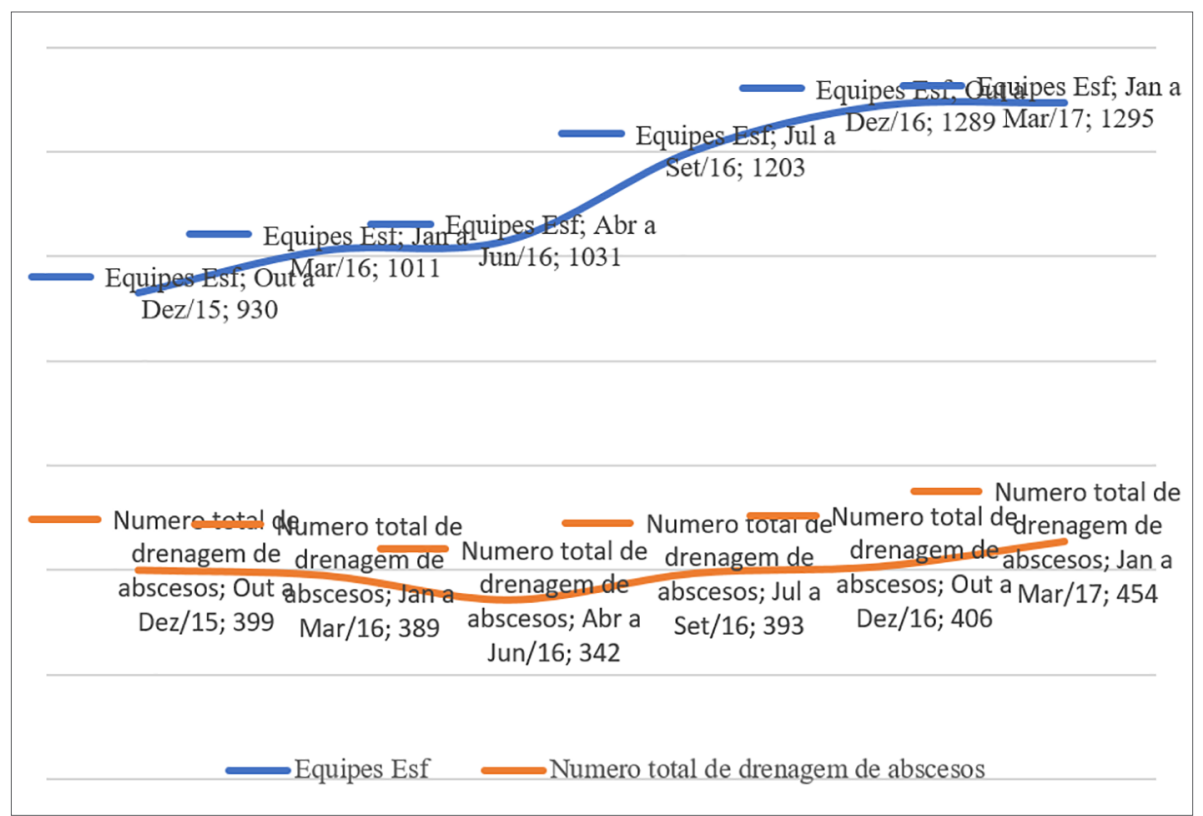

Gráfico 3. Distribuição dos registros de drenagem de abscessos existente no Carteirômetro em relação ao número de equipes por trimestre.

Fonte. Carteirometro/SUBPAV, 2017. Org. Oliveira, 2017.

A variação negativa em termos absolutos da realização de cantoplastia (Gráfico 4) pode estar relacionada ao fato de esta ser uma intervenção que exige habilidades cirúrgicas específicas, às quais a maioria dos médicos pode não ter sido treinada na graduação médica ou em estágios em unidades de emergência para fazer esta intervenção.

Os dados extraídos das plataformas Carteirômetro fornecem um retrato da realização de cirurgia ambulatorial na APS nos 18 meses estudados. A expansão da ESF levou ao incremento em números absolutos destes procedimentos. Contudo, estes resultados estão aquém da capacidade da APS, principalmente quando discutimos sobre uma APS abrangente, qualificada e resolutiva, capaz de organizar as redes de atenção à saúde.

Dos 1263 questionários enviados aos médicos atuantes na APS da cidade, apenas 69 (5,5\%) médicos responderam. Uma taxa de resposta abaixo daquela estimada na literatura, que é entre 7\% e $13 \%$. Porém, este menor número alcançado não inviabilizou a possibilidade de uma análise exploratória sobre as condições e percepções dos MFC sobre suas práticas. 


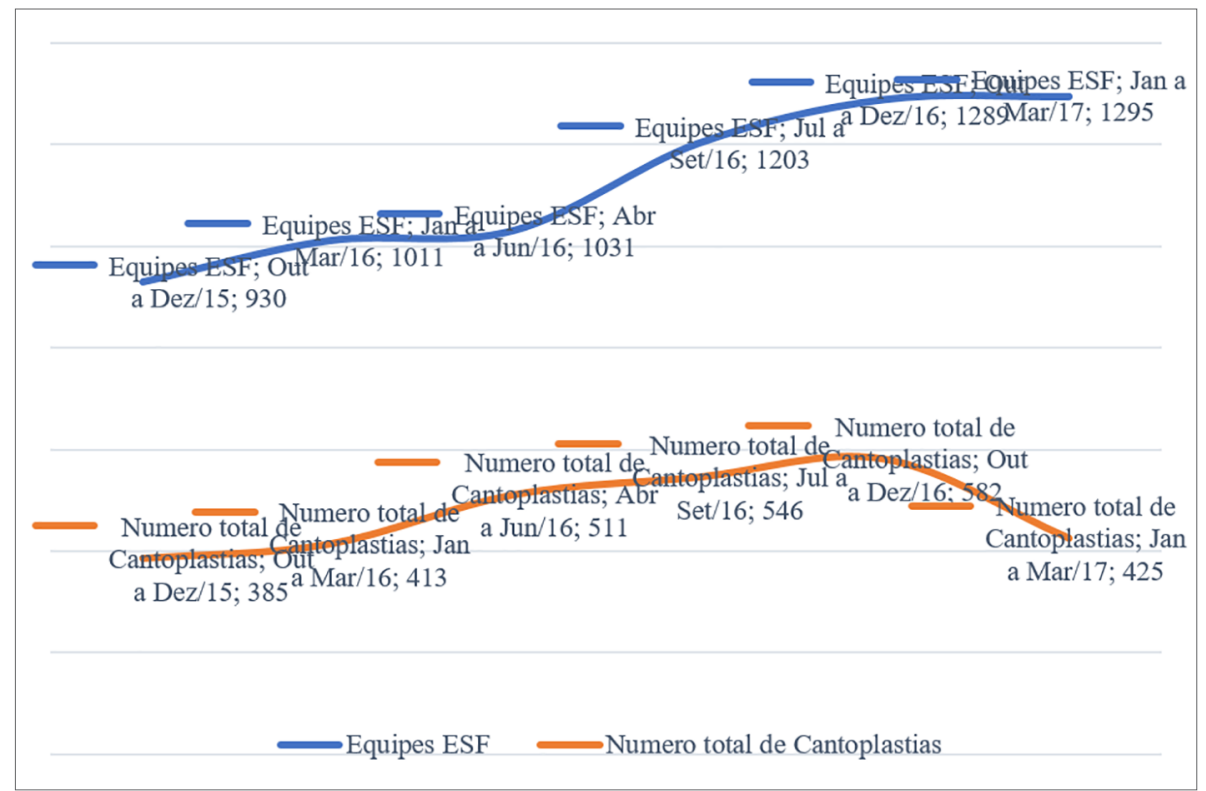

Gráfico 4. Distribuição dos registros de cantoplastia existente no Carteirômetro em relação ao número de equipes ESF por trimestre.

Fonte. Carteirometro/SUBPAV, 2017. Org. Oliveira, 2017.

Em relação ao perfil dos respondentes, $66 \%$ eram do sexo feminino, a maioria tinha entre 30 e 39 anos (50\%) e $76 \%$ dos profissionais eram formados há mais de 5 anos. A maioria dos médicos tinha menos de 10 anos de atuação na ESF, destes 32\% entre 2 e 5 anos, e $48 \%$ entre 6 a 10 anos.

Quanto à realização de especialização (residência ou título de especialista), a grande maioria (83\%) afirmou ter realizado especialização em MFC. Este dado não corresponde à lotação de médicos especialistas na rede de atenção primária na cidade, onde a grande maioria não tem qualquer especialização em APS/ESF/MFC. ${ }^{12}$

O fato de que a grande maioria dos que atenderam à solicitação serem especialistas pode estar relacionado ao alto grau de positividade em relação à realização de algum dos procedimentos cirúrgicos listados $(90 \%)$ e de que apenas seis médicos (10\%) declararem não realizar qualquer intervenção. Este perfil de médicos especialistas pode representar que houve um viés de seleção dos respondentes ao questionário.

A quase totalidade dos entrevistados realizava drenagem de abscesso. Tal informação não corresponde aos dados encontrados nos registros do Carteirômetro. Estas respostas podem se relacionar a esta ser uma intervenção muito comum na prática clínica em geral e na formação médica, sem necessidade de qualificação ou condições físicas e materiais específicas. Por outro lado, menos da metade dos médicos afirmaram executar biópsia e exérese de lesões suspeitas de pele, as quais envolvem necessidade de maior capacitação profissional e de fluxos para diagnóstico histopatológico.

A maioria dos respondentes (65\%) não identificou impedimento para executar qualquer dos procedimentos cirurgicos listados. Este é um número compatível com o quantitativo de $70 \%$ que reconheciam como adequadas as condições estruturais para a realização das cirurgias.

Entre os que citaram não realizar um ou mais procedimentos cirúrgicos, as justificativas foram a ausência de condições estruturais nas unidades e a falta de capacitação, ambas com frequências 
semelhantes de resposta. A realização de histopatológico foi citada como uma dificuldade estrutural frequente, seguida pela ausência de materiais cirúrgicos adequados. Importante ressaltar que, dentre os seis entrevistados que não realizaram procedimentos no último ano, cinco identificaram a ausência de capacitação como justificativa.

A capacitação profissional específica para realizar cirurgias foi outro elemento apontado como limitador na realização de algumas das intervenções listadas. Um quantitativo elevado (45\%), embora tenha realizado algum tipo de procedimento, não participou de nenhuma capacitação. Apenas 24 entrevistados participaram de algum tipo de capacitacão e, destes, apenas um teve oportunidade fora do programa de residência de medicina de família (RMFC). A baixa ocorrência de treinamento em cirurgia ambulatorial pode ser uma explicação para o menor número de médicos que realizam procedimentos que exigem maior habilidade e segurança em técnicas cirúrgicas como a cantoplastia e biópsia de pele.

Ademais, 37 entrevistados afirmaram não haver, em suas unidades, um fluxo assistencial específico para a realização de cirurgias. Entre estes médicos, 15 citaram a falta de apoio da gestão e/ou pressão pela demanda assistencial como fatores que dificultam a realização dos procedimentos.

A maioria dos entrevistados (94\%) consideraram que a realização das cirurgias modifica positivamente a visão do usuário sobre a atenção prestada na APS. Ao especificarem o motivo desta mudança, $78 \%$ dos médicos relacionam a mudança na percepção dos pacientes quanto à resolutividade e, consequentemente, à qualidade da ESF em relação a modelos de atenção básica anteriores. Por fim, para 92,8\% dos entrevistados as práticas cirúrgicas contribuíam para o estreitamento do vínculo entre usuários, as equipes e os médicos.

\section{Discussão}

Na perspectiva de desenvolver uma APS abrangente, qualificada e resolutiva no município, observou-se a crescente incorporação de procedimentos de cirurgia ambulatorial pelas equipes da ESF.

A heterogeneidade no registro das informações dos procedimentos nos prontuários eletrônicos e nas nomenclaturas usadas no banco de dados do Carteirômetro pode influenciar o registro dos atendimentos/ procedimentos por parte dos profissionais e ter impactado nos resultados observados neste estudo.

Verificou-se nos dados do Carteirômetro que a expansão das equipes de ESF foi acompanhada do incremento de $69 \%$ no número absoluto de procedimentos realizados. No entanto, a relação de 1,5 procedimentos/equipe, no final do período estudado, evidenciou que, apesar do esforço de padronização e incentivo da carteira de serviços, ainda há uma baixa difusão da cirurgia ambulatorial na ESF. Logo, uma condição ainda insuficiente para reduzir a fila de usuários cadastrados no SISREG que aguardam atenção no nível secundário.

A taxa de resposta aos questionários online abaixo do estimado na lliteratura pode ser reflexo de dificuldades de ser uma pesquisa a distância ou de medo com possíveis cobranças profissionais. Porém, também pode significar que a grande maioria dos médicos ainda estão distantes deste tema e destas ações em seu trabalho na ESF.

Esta última impressão se baseia no fato de que a grande maioria dos que responderam tinham especialização em MFC/ESF. Consequentemente, maior interesse, conhecimento e sensibilização em relação à cirurgia ambulatorial no cotidiano de seus serviços. 
As informações dos questionários online apontaram que os procedimentos mais realizados nas unidades de saúde foram aqueles que exigem menor habilidade técnica, como é o caso de suturas e da drenagem de abscesso. Estes últimos, além de necessitarem de menor capacitação, exigem estruturas físicas e equipamentos mais simples das unidades de saúde. Este fato pode estar relacionado à velocidade acelerada de implantação de novas equipes em um curto período de tempo, sem ter havido possibilidade para qualificar os profissionais e adequação das condições das unidades de saúde de modo a permitir condições que viabilizassem a incorporação destes procedimentos.

A baixa ocorrência de treinamento em cirurgia ambulatorial, mesmo entre aqueles que afirmam serem especialistas em MFC, pode ser uma explicação para o menor número de médicos que realizam procedimentos que exigem maior habilidade e segurança em técnicas cirúrgicas como a cantoplastia e biópsia de pele.

Se por um lado a residência de MFC teve o papel preponderante como estratégia de capacitação para pequenas cirurgias, por outro, ela representa uma visão mais abrangente da APS por parte destes especialistas, o que pode ter relação com suas preocupações em ampliar o escopo e a resolutividade de suas práticas. Em um contexto no qual a grande maioria dos médicos atuantes não fez RMFC, percebeu-se a necessidade de que haja capacitação em cirurgia ambulatorial para os médicos da rede municipal.

A capacitação é fator importante para a realização de procedimentos de cirurgia ambulatorial. De acordo com Williams et al., ${ }^{15}$ é essencial que o General Practioner (GP) seja adequadamente capacitado para realizar estes procedimentos. Estudos, como o de George et al., ${ }^{16}$ questionam a eficácia da exérese de lesões de pele suspeitas de malignidade, justamente pela inabilidade dos GP em identificar e retirar de forma adequada (com margens de segurança) estas lesões, necessitando, muitas vezes, de novos procedimentos cirúrgicos em serviços de atenção secundária, o que eleva os custos.

Gervas et al., ${ }^{17}$ contudo, a partir do contexto do GP, advertem sobre a excessiva especialização e do risco de fragmentar a APS e surgimento de novos médicos especialistas em seu interior.

A maioria dos médicos respondentes percebe que os procedimentos de cirurgia ambulatorial na APS podem contribuir no acesso e integralidade do cuidado, fortalecer o vínculo dos usuários com as unidades e suas equipes e ampliar a resolutividade e qualificação da Rede de Atenção.

Observa-se que a APS possui uma potencialidade pouco explorada no Rio de Janeiro, nosso cenário de estudo, fato que pode se repetir no Brasil. A real compreensão desta potencialidade pode permitir um fortalecimento da APS, ainda muito fragilizada.

\section{Conclusão}

Este trabalho se propôs a delinear o quanto e como vem ocorrendo a incorporação da cirurgia ambulatorial na APS da cidade do Rio de Janeiro. Para traçar este perfil, foram explorados os dados secundários provenientes de bases de dados locais e consultados os profissionais médicos sobre suas práticas cirúrgicas na APS.

Compreende-se que, para viabilizar uma melhor avaliação desta produção de serviços, faz-se necessária a padronização, não só dos prontuários eletrônicos, mas também das nomenclaturas vigentes nos prontuários, assim como nos bancos de dados. 
A aplicação dos questionários online, embora seja uma ferramenta que facilita o tempo e os custos da pesquisa, trouxe limitações. Uma delas foi a pouca, e provavelmente seletiva, participação, pois o perfil dos respondentes tinha, majoritariamente, especialização em saúde da família ou residência em MFC. Por outro lado, esta seletividade pode ser um indicador de como a incorporação destes procedimentos na APS pode ter caráter diferenciado entre os médicos especialistas e não especialistas inseridos na ESF. Um indicador de maior envolvimento dos profissionais especializados na incorporação de novas práticas e na qualificação do trabalho da APS.

Conclui-se que a incorporação de novas tecnologias, entre elas a cirurgia ambulatorial, como um elemento no desenvolvimento de uma APS abrangente e qualificada, é importante para a organização da rede de atenção em saúde. Porém, este desenvolvimento é dependente de um crescente investimento em recursos financeiros e em capacitação dos profissionais.

A falta de investimentos na incorporação de práticas cirúrgicas na APS, o não reconhecimento destas práticas como parte das atribuições das equipes ESF, bem como do seu papel na garantia dos atributos essenciais da APS e na organização da RAS, pode estar refletida na carência de estudos sobre os procedimentos de cirurgia ambulatorial na atenção primária no Brasil. Esta foi a lacuna a qual este estudo pretendeu contribuir na perspectiva de desenvolvimento de uma APS de qualidade e acessível no nosso país.

\section{Contribuição dos autores}

Coleta e análise dos dados, escrita e desenvolvimento do texto do artigo: PRO. Análise dos dados, escrita e desenvolvimento do texto do artigo: CAOF.

\section{Conflito de interesses}

Declaram não haver.

\section{Referências}

1. Giovanella L, Escorel S, Lobato LVC, Noronha JC, Carvalho AI. Políticas e sistemas de Saúde no Brasil. $2^{\underline{a}}$ ed. Rio de Janeiro: Fiocruz; 2012.

2. Brasil. Ministério da Saúde. Secretaria de Atenção à Saúde. Departamento de Atenção Básica. Memórias da Saúde da Família. Brasília: Ministério da Saúde; 2010 [Internet]. [acesso 2016 Ago 15] Disponível em: bvsms.saude.gov.br/bvs/publicacoes/memorias_saude_ familia_brasil.pdf

3. Mendes EV. As Redes de Atenção à Saúde. 2ª ed. Brasília: Organização Pan-Americana da Saúde; 2011.

4. Starfield B. Atenção primária: equilíbrio entre necessidades de saúde, serviços e tecnologia. Brasília: UNESCO/Ministério da Saúde; 2002 [Internet]. [acesso 2016 Jul 17]. Disponível em: https://www.nescon.medicina.ufmg.br/biblioteca/imagem/0253.pdf

5. Rio de Janeiro. Secretaria Municipal de Saúde do Município do Rio de Janeiro. Subsecretária de Atenção Primária. Vigilância e Promoção da Saúde. Rio de Janeiro: Prefeitura Municipal do Rio de Janeiro; 2014. [Internet] [acesso 2017 Fev 24]. Disponível em: https://subpav. org/index.php

6. Rio de Janeiro. Secretaria Municipal de Saúde do Município do Rio de Janeiro. Plano Municipal de Saúde do Rio de Janeiro 20142017. Rio de Janeiro: Prefeitura Municipal do Rio de Janeiro; 2017 [Internet]. [acesso 2016 Abr 26]. Disponível em: www.rio.rj.gov.br/ dlstatic/10112/3700816/4128745/PMS_20142017.pdf 
7. Rio de Janeiro. Secretaria Municipal de Saúde e Defesa Civil (SMSDC). Superintendência de Atenção Primária. Carteira de serviços prestados na Atenção Primária. Rio de Janeiro: Prefeitura Municipal do Rio de Janeiro; 2011 [Internet]. [acesso 2016 Abr 26]. Disponível em: http://www.rio.rj.gov.br/dlstatic/10112/137240/DLFE-228987.pdf/1.0

8. Brasil. Ministério da Saúde. Sistema Nacional de Regulação. 2017 [Internet]. [acesso 2016 Ago 14]. Disponível em: http://sisregiii.saude. gov.br/

9. Piovesan A, Temporini ER. Pesquisa exploratória: procedimento metodológico para o estudo de fatores humanos no campo da saúde pública. Rev Saúde Pública. 1995;29(4):318-25. [acesso 2016 Jul 5]. Disponível em: http://www.scielo.br/scielo.php?pid=S0034$89101995000400010 \&$ script=sci_abstract\&tlng=pt

10. Brasil. Ministério da Saúde. Departamento de Atenção Básica. Procedimentos. Cadernos de Atenção Primária n. 30. Brasília: Ministério da Saúde; 2011. [Internet]. [acesso 2016 Mar 15]. Disponível em: http://dab.saude.gov.br/portaldab/biblioteca.php?conteudo=publicacoes/ cab30

11. Sociedade Brasileira de Medicina de Família e Comunidade. Currículo Baseado em Competências para Medicina de Família e Comunidade. 2015 [Internet]. [acesso 2016 Abr 23]. Disponível em:https://www.ufrgs.br/telessauders/documentos/biblioteca_em_saude/006_material_ saude_curriculo_competencias_mfc.pdf

12. Rio de Janeiro. Prefeitura Municipal do Rio de Janeiro. Plataforma SUBPAV. Rio de Janeiro: Prefeitura Municipal do Rio de Janeiro; 2017 [Internet]. [acesso 2017 Fev 2]. Disponível em: https://subpav.org/logar.php

13. Freitas $\mathrm{H}$, Janissek-Muniz R, Moscarola J. Dinâmica do processo de coleta e análise de dados via web. Congresso Internacional de Pesquisa Qualitativa (CIBRAPEQ). Taubaté: Congresso Internacional de Pesquisa Qualitativa;2004. Disponível em: http://gianti.ea.ufrgs. br/files/artigos/2004/2004_157_CIBRAPEQ.pdf

14. Bardin L. Análise de Conteúdo. São Paulo: Edições 70; 2016.

15. Williams RB, Burdge AH, Jones SL. Skin biopsy in general practice. BMJ. 1991;303(6811):1179-80. http://dx.doi.org/10.1136/ bmj.303.6811.1179

16. George S, Pockney P, Primrose J, Smith H, Little P, Kinley H, et al. A prospective randomised comparison of minor surgery in primary and secondary care. The MiSTIC trial. Health Technol Assess. 2008;12(23):iii-iv,ix-38.

17. Gérvas J, Starfield B, Violán C, Minué S. GPs with special interests: unanswered questions. Br J Gen Pract. 2007;57(544):912-7. http://dx.doi.org/10.3399/096016407782317865 